\title{
Estimativa e espacialização das temperaturas do ar mínimas, médias e máximas na Região Nordeste do Brasil
}

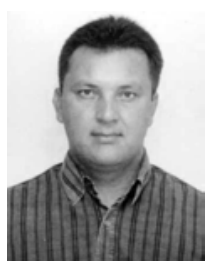

\author{
Salomão de S. Medeiros ${ }^{1}$, Roberto A. Cecílio ${ }^{1}$, Júlio C. F. de Melo Júnior ${ }^{1}$ \& José L. C. da Silva Junior ${ }^{1}$ \\ 1 DEA/UFV, CEP 36571-000, Viçosa, MG. Fone: (031) 3899 1939. E-mail: salommao@gmail.com (Foto); \\ rcecilio@vicosa.ufv.br; jcfmelojunior@bol.com.br; jlcabral@alunos.ufv.br
}

Protocolo $1-8 / 1 / 2004$ - Aprovado em 14/1/2005

\begin{abstract}
Resumo: Neste trabalho teve-se como objetivo principal, gerar equações de regressão múltiplas com ajuste de superfície quadrática para estimativa das normais das temperaturas do ar mínimas, médias e máximas mensais e anual, para o Nordeste brasileiro, a fim de fornecer subsídios para o planejamento agroclimático em regiões onde não há disponibilidade desses dados. Obtiveramse os dados de temperatura do ar utilizados para a determinação dos coeficientes das equações, de 74 estações meteorológicas listadas nas normais climatológicas dos Estados da região Nordeste, fornecidas pelo INMET. A partir das equações ajustadas, foram gerados mapas temáticos das normais das temperaturas do ar mínimas, médias e máximas mensais e anual. As equações obtidas apresentaram bons valores de $\mathrm{R}^{2}$; entretanto, quando utilizadas para altitudes muito elevadas, essas equações tenderam a apresentar baixos valores de temperatura do ar, enquanto as equações ajustadas e sua espacialização mostraram tratar-se de uma alternativa viável para a ampliação da base de dados climáticos da região Nordeste.
\end{abstract}

Palavras-chave: temperatura, equações de regressão, planejamento agroclimático

\section{Estimation and spatialization of minimum, mean and maximum air temperatures for the Northeast region of Brazil}

\begin{abstract}
This study aimed to generate multiple regression equations with quadratic functions to estimate both monthly and annual minimum, mean and maximum air temperatures for the Northeast region. It will provide subsidy to agroclimatic planning in the regions where these data are not available. Temperature data used to determine the equations coefficients were obtained from 74 meteorological stations listed in climatological normals of the states of Northeast region - Brazil, provided by INMET. Based on the adjusted models, thematic maps of monthly and annual minimum, mean and maximum air temperatures were generated. The obtained equations presented good values of $R^{2}$. However, when the equations were used for high altitude places they tended to present low values of air temperature. The adjusted equations and its spatialization is an alternative available to enlarge the climatic data base of this region.
\end{abstract}

Key words: temperature, regression equations, agroclimatic planning

\section{INTRODUÇÃO}

A temperatura do ar é, dentre os elementos climáticos, o que promove maiores efeitos diretos e significativos sobre muitos processos fisiológicos que ocorrem em plantas e animais; portanto, seu conhecimento se torna fundamental em estudos de planejamento agrícola e em análises de adaptação de culturas a determinadas regiões com características distintas.

Nos mapeamentos de campos homogêneos dos elementos do clima, a utilização de equações lineares cujas variáveis envolvem os fatores geográficos facilita, em muito, as definições das regiões limítrofes desses campos; além disso, a representatividade dos campos se torna mais próxima da realidade, uma vez que as cartas temáticas podem ter, como base de dados, o mesmo sistema de georreferenciamento. Os mapas de regionalização das informações das temperaturas médias, conhecendo-se as equações que representam as normais mensais e anual, possibilita analisar-se a abrangência geográfica dos campos homogêneos, apresentando os valores de temperatura em forma de mapas georreferenciados. A construção desses mapas da temperatura média normal georreferenciada é de enorme importância na interpolação e definição dos limites 
das regiões climaticamente homogêneas (Sediyama \& Melo Júnior, 1998; Sediyama et al., 2002).

O ajuste de equações de regressão múltipla tem possibilitado que se estime os valores médios mensais e anual das temperaturas mínimas, médias e máximas, em função da longitude, da latitude e da altitude; esta metodologia é bastante prática e tem apresentado um grau aceitável de acurácia. A estimativa das temperaturas do ar mínimas, médias e máximas mensais e anual a partir das coordenadas geográficas foi alvo de diversos estudos em diferentes estados e regiões do Brasil (Ferreira et al., 1971; Coelho et al., 1973; Ferreira et al., 1974; Feitoza et al., 1979; Camargo \& Ghizzi, 1991; Pedro Júnior et al., 1991; Cavalcanti \& Silva, 1994; Luiz \& Silva, 1995; Lima \& Ribeiro, 1998; Sediyama \& Melo Júnior, 1998; Sediyama et al., 2002; Marin et al., 2003).

Em zoneamentos de aptidão climática das culturas, por exemplo, as informações das condições térmicas regionais são elementos imprescindíveis e sua escassez sobre os dados meteorológicos em grandes áreas limita, muitas vezes, estudos suficientemente detalhados sobre os tipos climáticos de grande parte do território nacional (Sediyama \& Melo Júnior, 1998). O Nordeste brasileiro se depara com esta situação, em que a falta e a má distribuição de estações meteorológicas, inclusive naquelas onde a agricultura e a pecuária se configuram como principais atividades econômicas, tornando inviável o planejamento agropecuário.

Lima \& Ribeiro (1998), utilizaram equações lineares para estimativa das temperaturas do ar mínimas, médias e máximas mensais para o Estado do Piauí, em função da latitude, longitude e altitude local e obtiveram coeficientes de determinação ajustados que variaram entre 0,29 (temperatura máxima de fevereiro) a 0,84 (temperatura mínima de fevereiro). Marin et al. (2003) apresentaram equações de ajuste linear para estimativa da temperatura do ar no Nordeste, utilizando dados de 75 estações climatológicas do INMET, obtendo coeficientes de determinação variando entre 0,49 a 0,78 ; entretanto, os coeficientes foram maiores quando se desconsiderou a longitude das estações (Brasil, 1992).

Cavalcanti \& Silva (1994) apresentaram equações para estimativa da temperatura do ar em três sub-regiões do Nordeste: a) Maranhão e Piauí; b) Ceará, Rio Grande do Norte, Paraíba e Pernambuco; c) Alagoas, Sergipe e Bahia. Os coeficientes de correlação variaram de 0,70 a 0,99 . Utilizou-se a função de ajuste de superfície quadrática, em que a temperatura do ar é dada em função das longitude, latitude e altitude, e seus respectivos quadrados e produtos cruzados. Apesar dessas equações apresentarem maiores coeficientes de correlação que aqueles obtidos com o emprego de equações lineares não apresentam, porém, estimativas confiáveis quando extrapoladas fora dos limites das coordenadas geográficas.

Com base no exposto, os objetivos para o desenvolvimento deste trabalho foram:

- obter equações de regressão múltipla, com ajuste quadrático, para estimativa das temperaturas do ar mínimas, médias e máximas, mensais e anual para a região Nordeste do Brasil;

- gerar mapas temáticos de temperatura do ar mínimas, médias e máximas, mensais e anual, por meio das equações obtidas.

\section{MATERIAL E MÉTODOS}

Os dados de temperatura do ar coletados em abrigos termométricos padrões, utilizados para determinar os coeficientes das equações, foram obtidos de 74 das 75 estações meteorológicas listadas nas normais climatológicas dos estados da região Nordeste brasileira, fornecidas pelo INMET (Brasil, 1992) e apresentadas na Figura 1; apenas a estação de Fernando de Noronha foi excluída, por se localizar fora da parte continental do País.

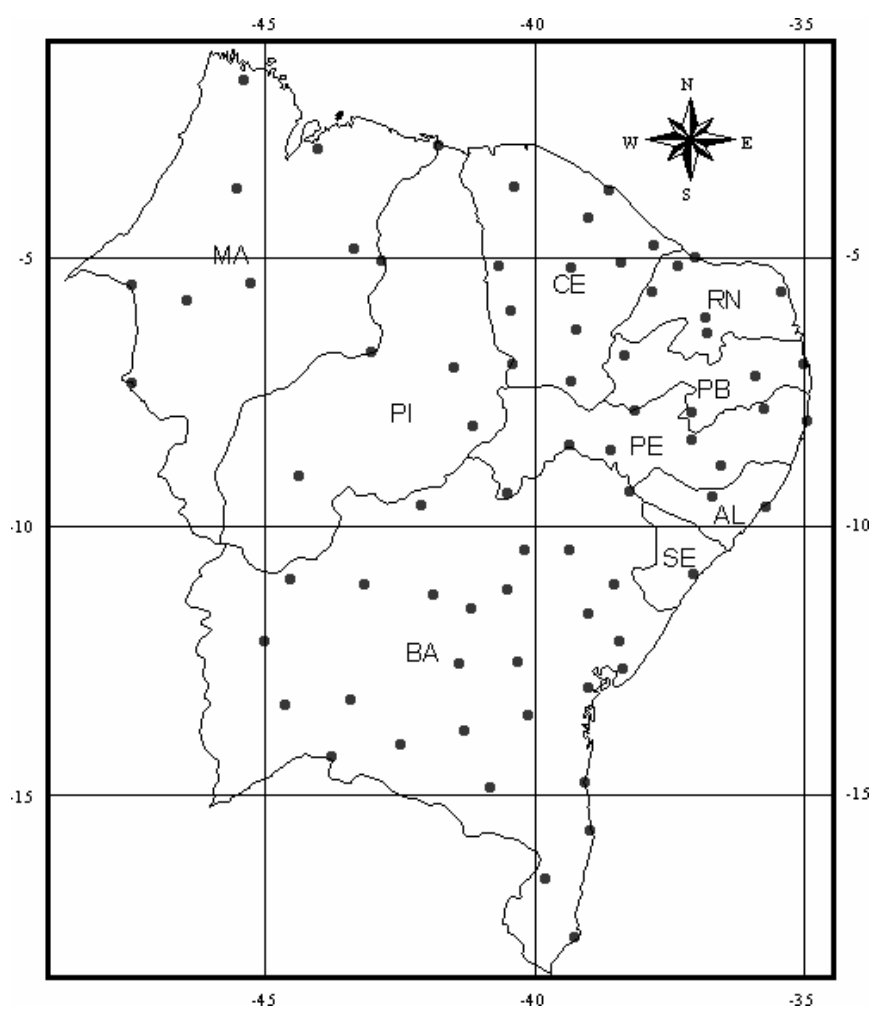

Figura 1. Distribuição espacial das estações meteorológicas da região Nordeste utilizadas no estudo

As análises de regressão múltipla foram efetuadas com os dados de temperaturas normais médias, máximas e mínimas, e anual, obedecendo ao seguinte modelo quadrático geral:

$$
\begin{gathered}
\mathrm{T}_{\mathrm{i}}=\mathrm{A}_{0}+\mathrm{A}_{1} \mathrm{~h}+\mathrm{A}_{2} \mathrm{~h}^{2}+\mathrm{A}_{3} \lambda+\mathrm{A}_{4} \lambda^{2}+\mathrm{A}_{5} \varphi+ \\
\mathrm{A}_{6} \varphi^{2}+\mathrm{A}_{7} \mathrm{~h} \varphi+\mathrm{A}_{8} \mathrm{~h} \lambda+\mathrm{A}_{9} \varphi \lambda
\end{gathered}
$$

em que:

$\mathrm{T}_{\mathrm{i}}$ - temperaturas normais (médias, máximas e mínimas) mensais $(i=1,2, \ldots, 12)$ e anual $(i=13)$ estimadas

$\lambda$ - longitude da estação (INMET) em graus e décimos (valores negativos)

$\phi$ - latitude da estação em graus e décimos (valores negativos)

$\mathrm{h}$ - altitude da estação em metros

$A_{n}$ - coeficientes da equação de regressão

As análises de regressão múltipla foram realizadas para todos os meses e também para o ano, sendo os coeficientes $\mathrm{A}_{\mathrm{n}}$ obtidos pelo método dos mínimos quadrados. 
Realizaram-se testes “t” de Student para se avaliar a significância dos coeficientes das equações de regressão $\left(A_{n}\right)$. Os termos cujos coeficientes não foram significativos a nível de $5 \%$ de probabilidade foram, no entanto, eliminados das equações. As equações obtidas pelas análises de regressão foram também avaliadas por intermédio do teste " $F$ " a nível de $5 \%$ de significância.

Com a finalidade de agrupar a região Nordeste em subregiões que possuam características homogêneas de temperatura do ar, realizou-se uma análise estatística multivariada de agrupamento considerando-se os valores normalizados de temperatura do ar de todas as estações meteorológicas da base de dados. Utilizou-se o método de agrupamento proposto por Ward (1963), com base na mínima e máxima variância intra e extraclasses, respectivamente, com o quadrado das distâncias euclidianas.

As temperaturas do ar foram espacializadas na forma de mapas temáticos, aplicando-se as equações de regressão às imagens digitais de latitude, longitude e altitude da região Nordeste, em um sistema de informações geográficas. Utilizouse, como mapa de altitude, o modelo digital de elevação, obtido por intermédio do United State Geological Survey, GTOPO 30, no formato matricial. O modelo digital de elevação possuía uma resolução espacial de aproximadamente um quilômetro quadrado, com dimensões dos atributos, em metros. Elaboraram-se mapas representativos das temperaturas do ar médias mensais e anual, utilizando-se as equações de regressão de superfície quadrática.

\section{RESULTADOS E DISCUSSÃO}

Com relação à análise de agrupamento em quatro grupos distintos, uma das classes de agrupamento mostrou-se completamente diversa das demais, sendo que as sete estações agrupadas nesta classe se encontraram dispersas na área analisada. Como o número de estações dessa classe foi menor que o número de termos da função quadrática, não se pode ajustar tal função à classe; portanto, a divisão do Nordeste em sub-regiões onde se esperava homogeneidade, foi descartada neste estudo; assim, as equações de regressão quadrática foram obtidas utilizando-se todas as 74 estações disponíveis, isto é, as regiões homogêneas de temperatura do ar não foram consideradas.

Os coeficientes das equações de regressão ajustadas para estimativas das temperaturas do ar média, máxima, mínima e anual, com os correspondentes coeficientes de determinação ajustados $\left(\mathrm{R}^{2}\right)$, são apresentados na Tabela 1 (A, B e C) respectivamente.

Os coeficientes de determinação ajustados das equações de regressão para se estimar os valores mensais e anual de temperatura do ar média, variaram de 0,86 (temperatura média do ar anual) a 0,67 (temperatura média do ar em fevereiro); para os valores mensais e anual de temperatura do ar máxima, oscilaram de 0,85 (temperatura máxima do ar em julho) a 0,52 (temperatura máxima do ar em fevereiro) e, para os valores mensais e anual de temperatura do ar mínima, de 0,85 (temperatura máxima do ar em abril) a 0,64 (temperatura máxima do ar em novembro).

Os mais baixos valores de $\mathrm{R}^{2}$ obtidos se devem, provavelmente, à grande variabilidade dos dados de temperatura do ar, nos meses em que estes ocorreram. De maneira geral, os valores de $\mathrm{R}^{2}$ tenderam a apresentar maiores valores para o ano que para meses, com exceção da temperatura máxima, que apresentou maiores valores de $\mathrm{R}^{2}$ entre abril e agosto.

Observou-se que, no mês de fevereiro, nas equações de estimativa da temperatura média e mínima do ar, dentre as variáveis geográficas consideradas e suas interações, apenas o fator altitude foi significativo. Constatou-se, também na Tabela 1(A, B e C), que a interação da altitude com a longitude não foi significativa para nenhuma das equações geradas.

Os valores de $\mathrm{R}^{2}$ referentes às equações para estimativa da temperatura do ar máxima apresentaram valores menores, visto que das 13 equações ajustadas para temperatura máxima do ar, cinco apresentaram valores menores que 0,70 , ocorrendo o mesmo também com três equações para estimativa da temperatura mínima do ar e com apenas um para a estimativa da temperatura média do ar.

As equações de regressão para estimativa das temperaturas média, máxima e mínima, propostas por Cavalcanti \& Silva (1994), para três sub-regiões do Nordeste brasileiro, apresentam maiores valores de $\mathrm{R}^{2}$ que as equações ajustadas no presente estudo mas, quando se procedeu à espacialização (Figura 2) das temperaturas do ar médias anuais geradas pelas equações propostas por Cavalcanti \& Silva (1994) e por este estudo, notou-se que a temperatura do ar na maior parte da sub-região do Maranhão e Piauí, é subestimada, chegando a apresentar temperaturas do ar inferiores a $10^{\circ} \mathrm{C}$ nas localidades de maiores altitudes. Tal fato mostra certa deficiência nas equações de Cavalcanti \& Silva (1994) quando aplicadas para a estimativa das temperaturas em locais de altitudes elevadas, pertencentes a esta sub-região, fato que não ocorre com as equações geradas neste trabalho.

Analisando-se a Figura 2A, percebe-se que o mapa não apresenta grandes descontinuidades espaciais na variação da temperatura do ar média anual, pelo fato da consideração de uma única equação se estender a toda à região. $\mathrm{O}$ mapa da Figura 2B, entretanto, mostra o contrário, ou seja: pelo fato de considerar três sub-regiões distintas e também todos os termos das equações significativos, acabou por causar descontinuidade no mapa de temperatura, mostrando que as equações não apresentam valores semelhantes em torno do limite entre sub-regiões, fato evidenciado por Valeriano \& Picini (2000) na espacialização de temperaturas do ar, para duas regiões distintas do estado de São Paulo.

A Figura 3 apresenta as diferenças médias percentuais (em termos relativos) entre os valores espacializados de temperatura do ar média anual, obtidos com a equação apresentada no presente estudo e o proposto por Cavalcanti \& Silva (1994). 
Tabela 1. Coeficientes das equações de regressão para estimativa dos valores mensais e anual de temperatura média, máxima e mínima do ar, com seus respectivos coeficientes de determinação ajustados $\left(\mathrm{R}^{2}\right)$ para a região Nordeste brasileira

\begin{tabular}{|c|c|c|c|c|c|c|c|c|c|c|c|}
\hline Mês & $\mathrm{R}^{2}$ & $\mathrm{~A}_{0}$ & $A_{1}$ & $\mathrm{~A}_{2}$ & $\mathrm{~A}_{3}$ & $\mathrm{~A}_{4}$ & $\mathrm{~A}_{5}$ & $\mathrm{~A}_{6}$ & $\mathrm{~A}_{7}$ & $\mathrm{~A}_{8}$ & $\mathrm{~A}_{9}$ \\
\hline \multicolumn{12}{|c|}{ A. Temperatura Média } \\
\hline Jan & 0,82 & $-25,99240$ & ns & $-0,000006$ & $-2,67684$ & $-0,03354$ & ns & $-0,00513$ & ns & ns & ns \\
\hline Fev & 0,67 & 26,34049 & ns & $-0,000006$ & ns & Ns & $\mathrm{ns}$ & ns & $\mathrm{ns}$ & $\mathrm{ns}$ & ns \\
\hline Mar & 0,77 & 26,32828 & $\mathrm{~ns}$ & $-0,000006$ & ns & Ns & ns & ns & ns & $\mathrm{ns}$ & $\mathrm{ns}$ \\
\hline Abr & 0,82 & 33,81864 & $-0,00589$ & ns & ns & $-0,00392$ & 2,04365 & $\mathrm{~ns}$ & $\mathrm{~ns}$ & $\mathrm{~ns}$ & 0,04897 \\
\hline Mai & 0,81 & 24,51485 & $-0,00620$ & ns & ns & ns & $-0,03856$ & ns & ns & $\mathrm{ns}$ & 0,01487 \\
\hline Jun & 0,81 & 24,46099 & $-0,00673$ & ns & ns & $\mathrm{ns}$ & ns & $-0,03910$ & ns & $\mathrm{ns}$ & 0,01339 \\
\hline Jul & 0,81 & 27,70850 & $-0,00640$ & ns & ns & ns & 1,48750 & ns & $\mathrm{ns}$ & $\mathrm{ns}$ & 0,02993 \\
\hline Ago & 0,83 & 36,16463 & ns & $\mathrm{ns}$ & ns & $-0,00459$ & 4,08316 & ns & 0,00017 & $\mathrm{~ns}$ & 0,09392 \\
\hline Set & 0,78 & 40,52614 & $-0,00696$ & ns & ns & $-0,00667$ & 4,83384 & $\mathrm{~ns}$ & ns & ns & 0,11274 \\
\hline Out & 0,72 & $-54,51334$ & ns & $-0,000006$ & $-3,87154$ & $-0,04503$ & ns & $-0,01407$ & ns & $\mathrm{ns}$ & ns \\
\hline Nov & 0,72 & $-68,59439$ & $\mathrm{~ns}$ & $-0,000006$ & $-4,68628$ & $-0,05634$ & $\mathrm{~ns}$ & $-0,01385$ & ns & $\mathrm{ns}$ & $\mathrm{ns}$ \\
\hline Dez & 0,74 & $-50,90658$ & ns & $-0,000006$ & $-3,86843$ & $-0,04736$ & ns & $-0,00996$ & ns & $\mathrm{ns}$ & ns \\
\hline Ano & 0,86 & 32,45762 & ns & $-0,000006$ & ns & $-0,00297$ & 1,87032 & ns & $\mathrm{ns}$ & ns & 0,04181 \\
\hline \multicolumn{12}{|c|}{ B. Temperatura Máxima } \\
\hline Jan & 0,61 & $-50,85409$ & ns & $-0,000006$ & $-4,16962$ & $-0,05330$ & ns & $-0,04664$ & ns & ns & 0,01803 \\
\hline Fev & 0,52 & 29,73803 & $\mathrm{~ns}$ & $-0,000005$ & ns & ns & $\mathrm{ns}$ & $-0,03078$ & $\mathrm{~ns}$ & $\mathrm{~ns}$ & 0,01373 \\
\hline Mar & 0,59 & 28,40990 & ns & $-0,000005$ & ns & $\mathrm{ns}$ & $\mathrm{ns}$ & $-0,03510$ & $\mathrm{~ns}$ & $\mathrm{~ns}$ & 0,01778 \\
\hline Abr & 0,78 & 36,22981 & ns & $-0,000007$ & ns & $-0,00407$ & 2,08949 & $-0,02890$ & ns & $\mathrm{ns}$ & 0,06367 \\
\hline Mai & 0,78 & 29,19504 & ns & $-0,000007$ & ns & $\mathrm{ns}$ & 1,26667 & $-0,03425$ & $\mathrm{~ns}$ & $\mathrm{~ns}$ & 0,04409 \\
\hline Jun & 0,83 & 41,70450 & $\mathrm{~ns}$ & $-0,000008$ & ns & $-0,00532$ & 4,62647 & ns & $\mathrm{ns}$ & $\mathrm{ns}$ & 0,10702 \\
\hline Jul & 0,85 & 42,64441 & ns & $-0,000008$ & ns & $-0,00546$ & 5,38557 & $\mathrm{~ns}$ & $\mathrm{~ns}$ & $\mathrm{~ns}$ & 0,12387 \\
\hline Ago & 0,81 & 48,03787 & $\mathrm{~ns}$ & $-0,000008$ & ns & $-0,00782$ & 6,58094 & $\mathrm{~ns}$ & $\mathrm{~ns}$ & $\mathrm{~ns}$ & 0,15190 \\
\hline Set & 0,75 & 50,16980 & ns & $-0,000007$ & $\mathrm{~ns}$ & $-0,00870$ & 6,78257 & ns & $\mathrm{ns}$ & $\mathrm{ns}$ & 0,15793 \\
\hline Out & 0,70 & $-124,94330$ & ns & $-0,000006$ & $-7,51507$ & $-0,09057$ & $\mathrm{~ns}$ & $-0,08315$ & ns & $\mathrm{ns}$ & 0,02915 \\
\hline Nov & 0,61 & $-100,84546$ & $\mathrm{~ns}$ & $-0,000006$ & $-6,48040$ & $-0,07929$ & $\mathrm{~ns}$ & $-0,06700$ & $\mathrm{~ns}$ & $\mathrm{~ns}$ & 0,02234 \\
\hline Dez & 0,67 & $-78,43840$ & 0,00703 & $-0,000012$ & $-5,50927$ & $-0,06770$ & $\mathrm{~ns}$ & $-0,01700$ & $\mathrm{~ns}$ & $\mathrm{~ns}$ & ns \\
\hline Ano & 0,77 & 39,53140 & ns & $-0,000007$ & ns & $-0,00503$ & 3,07006 & $-0,03200$ & $\mathrm{~ns}$ & $\mathrm{~ns}$ & 0,08526 \\
\hline \multicolumn{12}{|c|}{ C. Temperatura Mínima } \\
\hline Jan & 0,78 & 23,83374 & $-0,00544$ & $\mathrm{~ns}$ & $\mathrm{~ns}$ & ns & 0,10733 & ns & ns & ns & ns \\
\hline Fev & 0,79 & 23,10350 & $-0,00588$ & ns & ns & ns & $\mathrm{ns}$ & ns & ns & ns & ns \\
\hline Mar & 0,74 & 22,91020 & $-0,00545$ & ns & ns & $\mathrm{ns}$ & ns & ns & ns & ns & ns \\
\hline Abr & 0,85 & 23,30418 & $-0,00551$ & $\mathrm{~ns}$ & $\mathrm{~ns}$ & ns & $\mathrm{ns}$ & $-0,00635$ & ns & $\mathrm{ns}$ & $\mathrm{ns}$ \\
\hline Mai & 0,80 & 22,98452 & n.s. & ns & ns & ns & ns & $-0,00941$ & 0,00015 & ns & ns \\
\hline Jun & 0,70 & 23,20083 & $-0,00539$ & ns & ns & ns & ns & ns & ns & $\mathrm{ns}$ & $-0,00637$ \\
\hline Jul & 0,76 & 22,89712 & $-0,00582$ & $\mathrm{~ns}$ & ns & $\mathrm{ns}$ & $\mathrm{ns}$ & ns & ns & $\mathrm{ns}$ & $-0,00706$ \\
\hline Ago & 0,74 & 23,07974 & $-0,00648$ & ns & ns & ns & 0,25889 & ns & ns & ns & $\mathrm{ns}$ \\
\hline Set & 0,68 & 23,69265 & $-0,00612$ & ns & ns & ns & 0,22553 & ns & ns & $\mathrm{ns}$ & $\mathrm{ns}$ \\
\hline Out & 0,66 & 24,12035 & $-0,00544$ & ns & $\mathrm{ns}$ & $\mathrm{ns}$ & 0,19532 & ns & ns & $\mathrm{ns}$ & $\mathrm{ns}$ \\
\hline Nov & 0,64 & 24,03082 & $-0,00515$ & ns & $\mathrm{ns}$ & $\mathrm{ns}$ & ns & ns & ns & $\mathrm{ns}$ & $-0,00377$ \\
\hline Dez & 0,78 & $-34,44682$ & $-0,00545$ & ns & $-2,90031$ & $-0,03542$ & $\mathrm{~ns}$ & ns & ns & $\mathrm{ns}$ & $-0,00434$ \\
\hline Ano & 0,83 & 23,59970 & $-0,00552$ & $\mathrm{~ns}$ & ns & $\mathrm{ns}$ & ns & ns & ns & ns & $-0,00423$ \\
\hline
\end{tabular}

ns - Não significativo a nível de $5 \%$ de probabilidade, conforme o teste "t" de Student 
A.

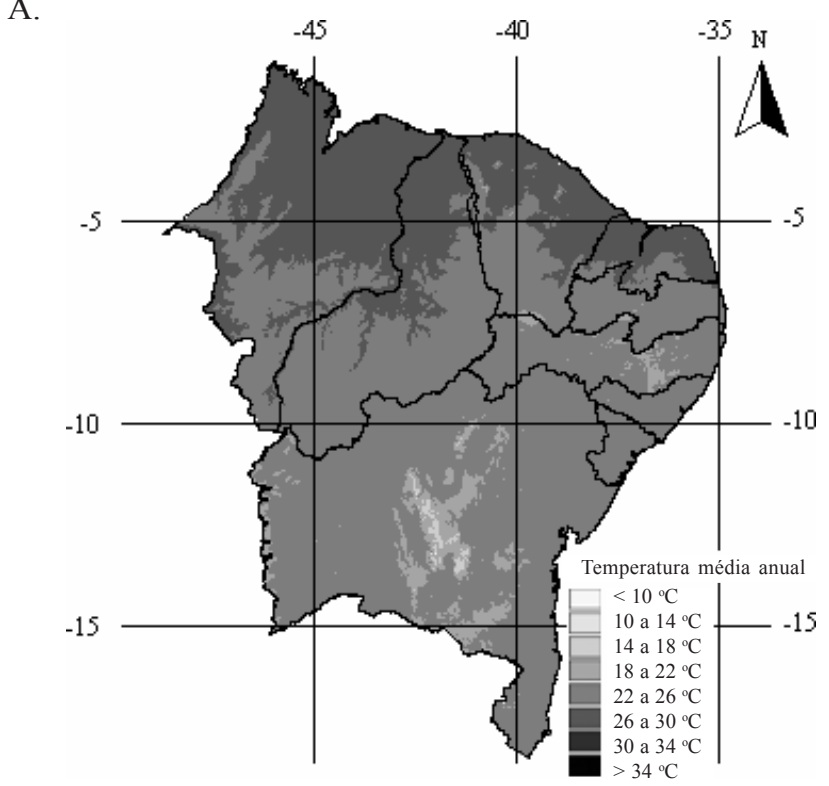

B.

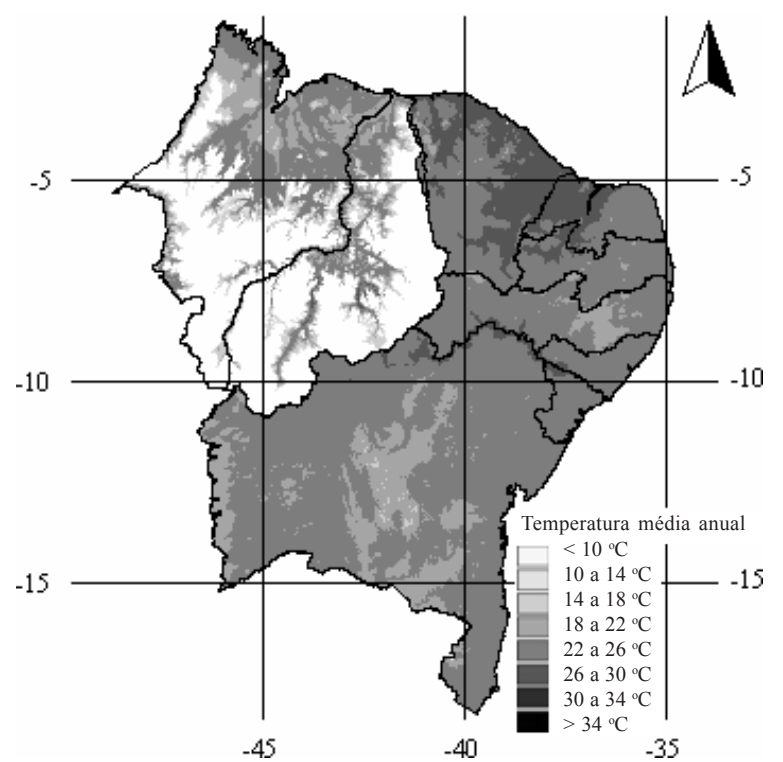

Figura 2. Espacialização da temperatura média anual para região Nordeste brasileira utilizando-se: (A) equação obtida no presente estudo e (B) equação proposta por Cavalcanti \& Silva (1994)

Constata-se, na Figura 3, que as diferenças percentuais relativas entre os valores estimados obtidos pela equação apresentada no presente estudo e a proposta por Cavalcanti \& Silva (1994) são muito pequenas, não atingindo $5 \%$ em duas subregiões do Nordeste: aquela constituída pelos estados do Ceará, Rio Grande do Norte, Paraíba e Pernambuco; e aquela composta por Alagoas, Sergipe e Bahia. Todavia, na sub-região dos estados do Maranhão e Piauí as diferenças variam de muito baixas na área mais próxima ao litoral a extremamente altas (maiores que 100\%) no interior dos estados, justamente onde as altitudes são maiores.

Nas Figuras 4, 5 e 6 são apresentados os mapas temáticos das estimativas das temperaturas do ar normais médias, máximas e mínimas mensais e anual, espacializados segundo as equações de regressão ajustadas.

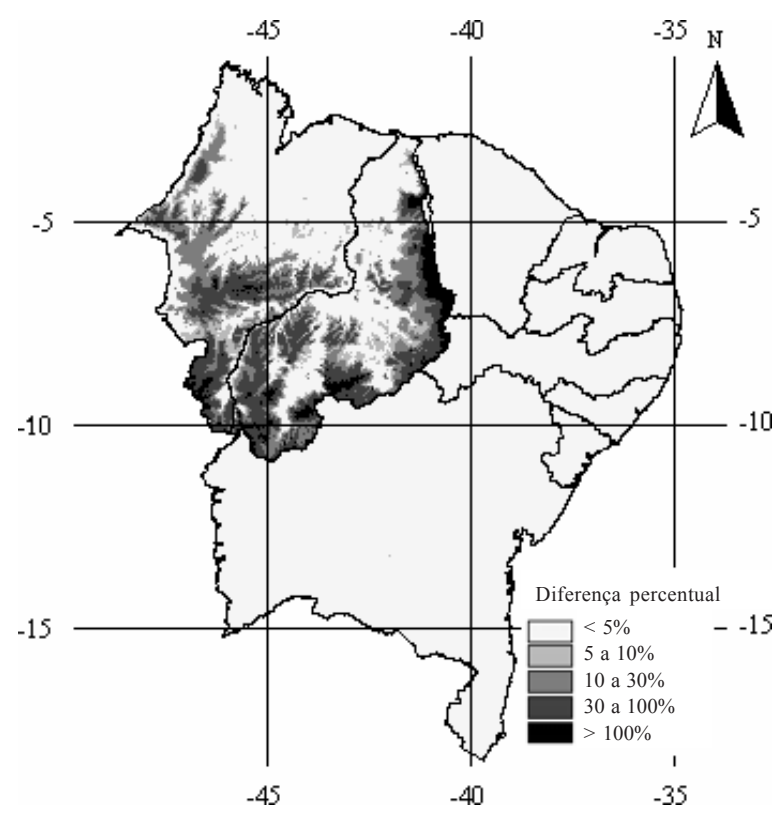

Figura 3. Diferenças médias percentuais entre valores espacializados de temperatura do ar média anual obtidos com a equação apresentada pelo presente estudo e o proposto por Cavalcanti \& Silva (1994)

Na Figura 4 observa-se que os meses de junho e julho apresentam as menores temperaturas do ar normais médias, com tendência de aumento a partir do mês de agosto.

$\mathrm{Na}$ maior parte da região Nordeste brasileira as temperaturas do ar normais médias mensais variam entre 18 e $26^{\circ} \mathrm{C}$, sendo que, em alguns meses, estas foram menores em pequenas áreas situadas na região central do estado da Bahia, e maiores nos estados do Maranhão, Piauí, Ceará e no norte do estado da Bahia.

De acordo com a Figura 5, em quase toda a região Nordeste brasileira as temperaturas do ar normais máximas mensais observadas foram superiores a $30^{\circ} \mathrm{C}$, com exceção de uma área situada ao extremo sul no estado da Bahia, na qual as temperaturas não atingiram este valor em nenhum mês do ano nem em algumas outras áreas isoladas, principalmente no centro deste Estado. A maior área dos estados do Maranhão e Piauí, além do Noroeste da Bahia, apresentou maiores valores para as temperaturas do ar normais máximas mensais.

De acordo com a Figura 6 verificou-se, na região Centro-Sul do Estado da Bahia, que as menores temperaturas do ar normais mínimas mensais, variaram de 10 a $14^{\circ} \mathrm{C}$ entre os meses de junho a setembro. Os maiores valores de temperatura do ar mínima foram verificados nas áreas situadas no extremo norte da região Nordeste, principalmente no litoral do estado do Maranhão.

Notou-se, pelos mapas temáticos (Figuras 4, 5 e 6) que a região central do Estado da Bahia apresentou, em todos os meses, menores temperaturas do ar normais médias, cujo fato se deveu às maiores altitudes dessas áreas, embora em alguns casos as altitudes aí encontradas tenham sido maiores que aquelas utilizadas no ajuste das equações de regressão, podendo haver subestimativa da temperatura normal média nesses locais. 

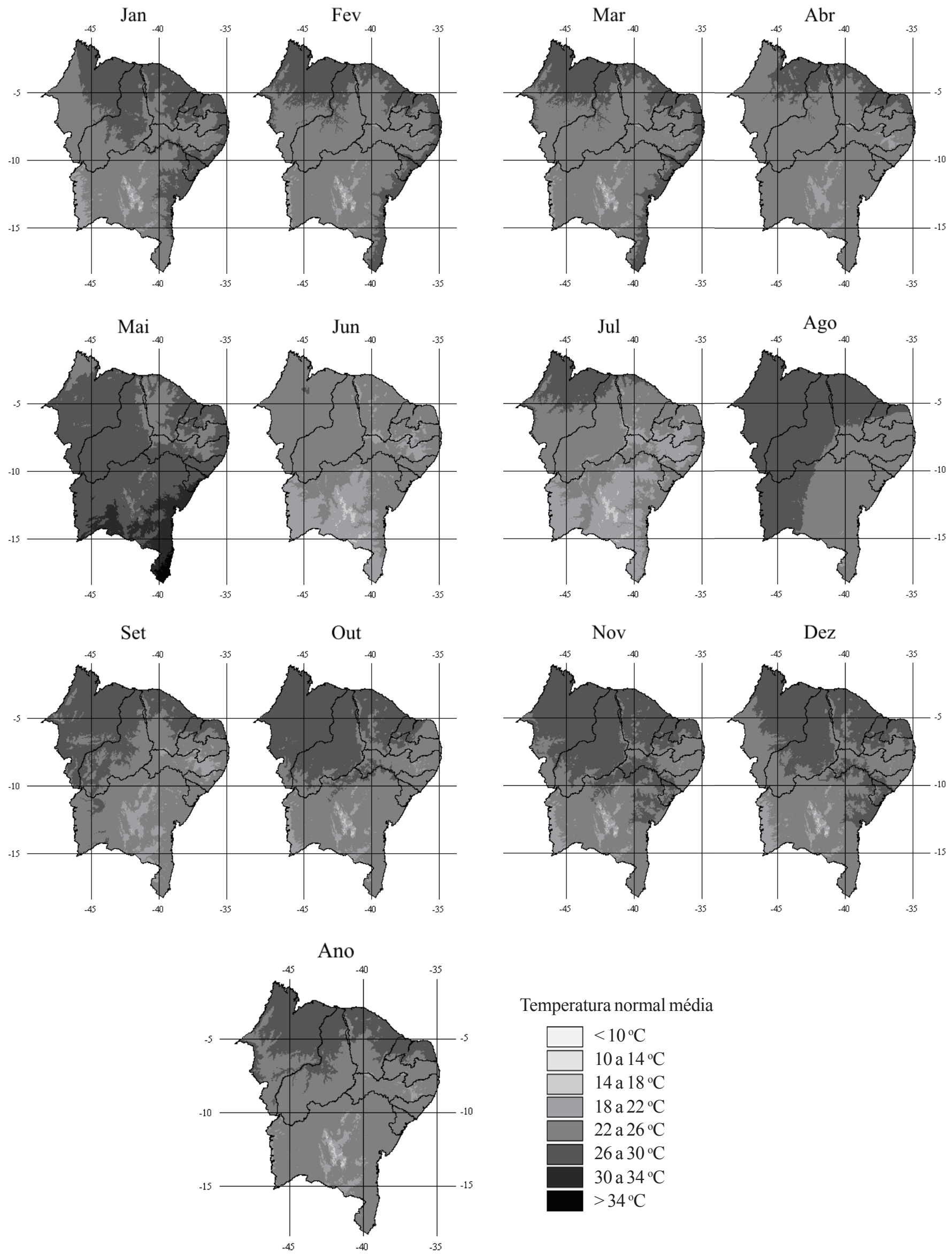

Figura 4. Mapas temáticos de temperaturas normais do ar média mensal e anual, espacializadas segundo as equações de regressão ajustadas 

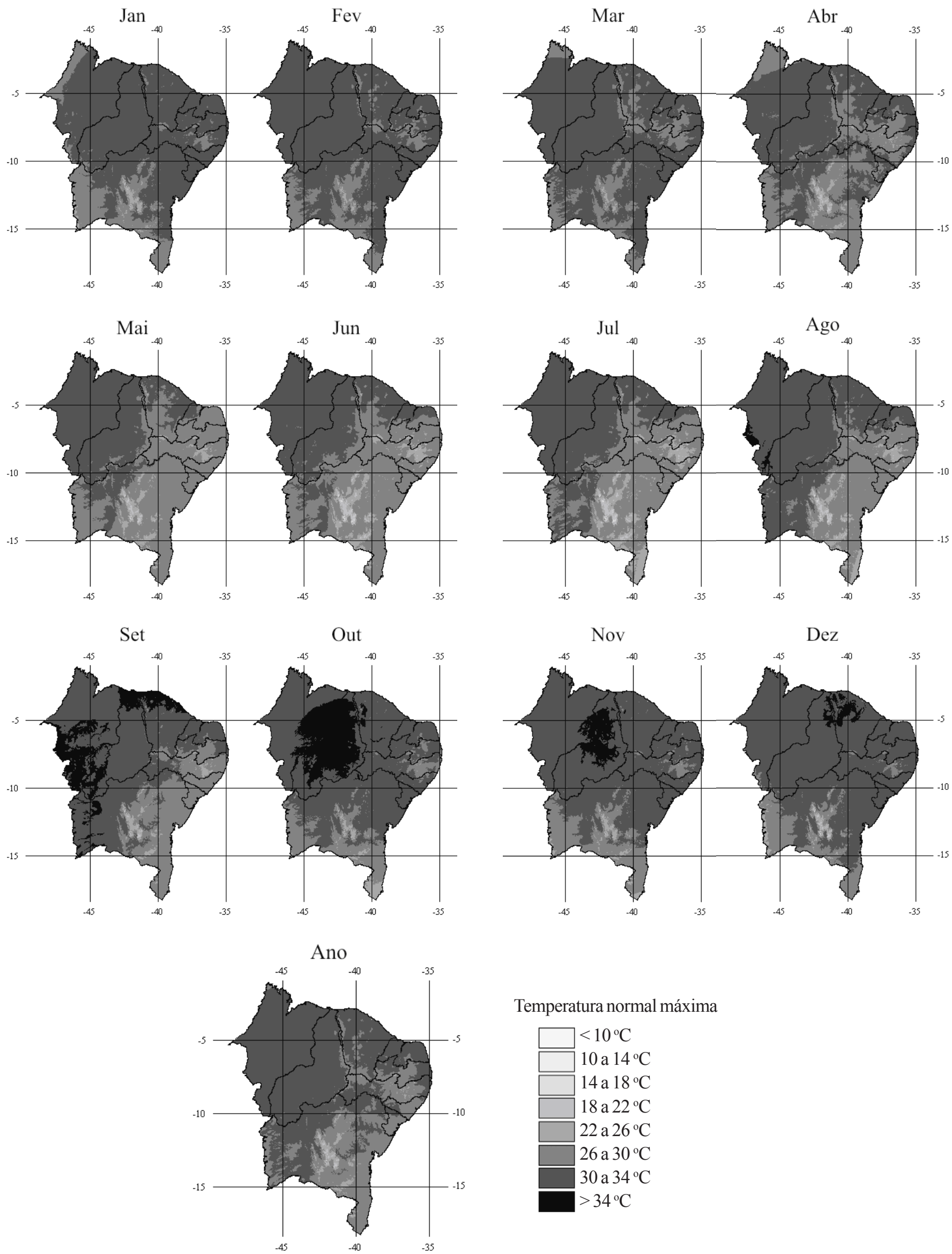

Temperatura normal máxima

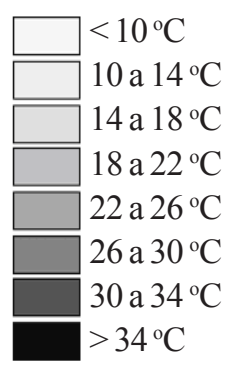

Figura 5. Mapas temáticos de temperaturas normais do ar máxima mensais e anual, espacializadas segundo as equações de regressão ajustadas 

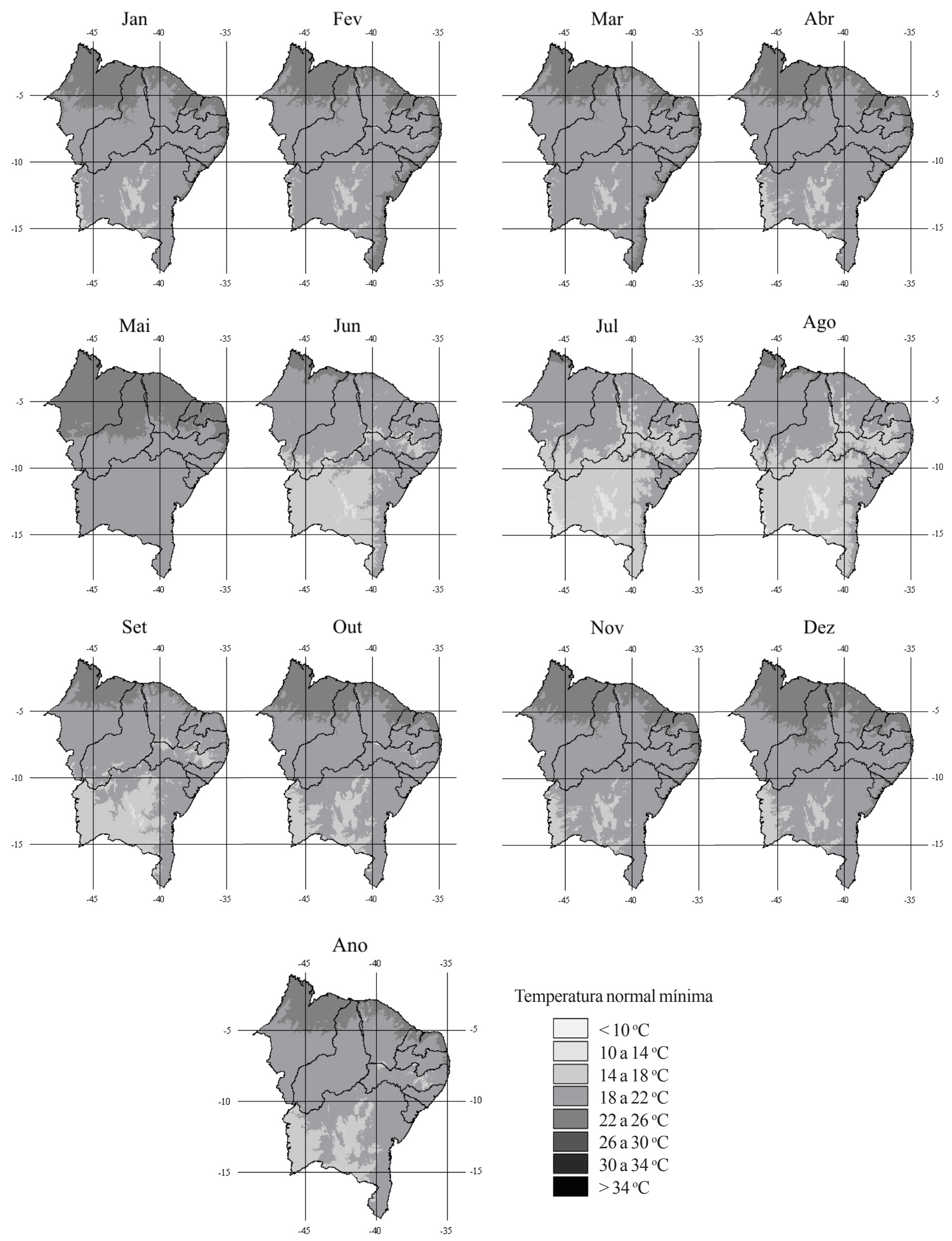

Figura 6. Mapas temáticos de temperaturas normais do ar mínimas mensais e anual, espacializadas segundo as equações de regressão ajustadas 


\section{CONCLUSÕES}

1. As equações ajustadas neste trabalho, considerando-se altitude, latitude e longitude do local, podem ser utilizadas para as estimativas das normais das temperaturas médias, máximas e mínimas mensais e anual, nos estados da região Nordeste brasileira.

2. A performance das equações obtidas neste estudo foi semelhante à daquelas equações obtidas na literatura para a mesma região, apresentando melhores resultados para os estados do Piauí e Maranhão.

3. O ajuste de equações de regressão para estimativa das normais das temperaturas mínimas, médias e máximas mensais e anual, é uma alternativa viável para ampliar a base de dados climáticos, uma vez que, a partir de 74 pontos de observações de temperatura do ar, foi possível elaborar mapas temáticos de temperatura do ar para toda a região Nordeste brasileira fornecendo, assim, subsídios para um melhor planejamento agropecuário da região.

\section{AGRADECIMENTOS}

Os autores agradecem às Empresas F. Medeiros Auto Peças Ltda e Socrauto pelo apoio financeiro e incentivo dispensados para o desenvolvimento deste trabalho; são gratos, também, à Universidade Federal de Viçosa - UFV, pelo apoio logístico.

\section{LITERATURA CITADA}

Brasil. Ministério da Agricultura e Reforma Agrária. Departamento Nacional de Meteorologia. Normais Climatológicas (1961-1990). Brasília: SPI/EMBRAPA, 1992. 84p.

Camargo, A.P.; Ghizzi, S.M. Estimativa das temperaturas médias com base em cartas de temperatura potencial normal ao nível do mar para a região sudeste do Brasil. Campinas, n.141, 1991. 17p. Boletim Técnico IAC

Cavalcanti, E.P.; Silva, E.D.V. Estimativa da temperatura do ar em função das coordenadas locais. In: Congresso Brasileiro de Meteorologia, 7, Congresso Latino-Americano e Ibérico de Meteorologia, 1994, Belo Horizonte. Anais..., Belo Horizonte: Sociedade Brasileira de Meteorologia, 1994, p.154157.

Coelho, D.T.; Sediyama, G.C.; Vieira, M. Estimativa das temperaturas médias mensais e anual no Estado de Minas Gerais. Revista Ceres, Viçosa, 20,112,455-459. 1973.
Feitoza, L.R.; Scárdua, J.A.; Sediyama, G.C.; Oliveira, L.M.; Valle, S.S. Estimativa das temperaturas médias mensais e anual do Estado do Espírito Santo. Revista Centro de Ciências Rurais, Santa Maria, v.9, n.3, p.279-291, 1979.

Ferreira, M.; Buriol, G.A.; Estefanel, V.; Pinto, H.S. Estimativa das temperaturas médias mensais e anuais do Estado do Rio Grande do Sul. Revista Centro Ciências Rurais, Santa Maria, v.1, n.4, p.21-52, 1971.

Ferreira, M.; Buriol, G.A.; Pignataro, I.A.B.; Estefanel, V. Estimativa das temperaturas médias mensais e anuais do Estado de Santa Catarina. Revista Centro Ciências Rurais, Santa Maria, v.4, n.1, p.19-38, 1974.

Lima, M.G.; Ribeiro, V.Q. Equações de estimativa da temperatura do ar para o Estado do Piauí. Revista Brasileira de Agrometeorologia, Santa Maria, v.6, n.2, p.221-227, 1998.

Luiz, A.J.B.; Silva, F.A.M. Temperaturas decendiais máxima, mínima e média, como função da latitude e altitude, em Goiás. In: Congresso Brasileiro de Agrometeorologia, 9, 1995, Campina Grande. Anais... Campina Grande: SBA, 1995. p.234235.

Marin, F.R.; Pandorfi, H.; Ferreira, A.S. Estimativa das temperaturas máximas, mínimas e médias mensais para o Brasil. In: Congresso Brasileiro de Agrometeorologia, 13., 2003 Santa Maria. Anais..., Santa Maria: SBA 2003. CD-ROM

Pedro Júnior, M.J.; Mello, M.H.A.; Ortolani, A.A. Alfonsi, R.R.; Sentelhas, P.C. Estimativa das temperaturas médias mensais, das máximas e das mínimas para o Estado de São Paulo. Campinas: IAC, 1991.11p. Boletim Técnico, n.142.

Sediyama, G.C.; Melo Júnior, J.C.F. Modelos para estimativas das temperaturas normais mensais médias, máximas, mínimas e anual no Estado de Minas Gerais. Revista Engenharia na Agricultura, Viçosa, v.6, n.1, p.57-61, 1998.

Sediyama, G.C.; Melo Júnior, J.C.F.; Santos, A.R.; Souza, J.A.; Santana, M.O. Modelo para estimativa das temperaturas normais mensais médias, máximas, mínimas e anual georreferenciados para o Estado do Espírito Santo. In: Congresso Brasileiro Engenharia Agrícola, 31, 2002, Salvador. Anais..., Salvador: Sociedade Brasileira de Engenharia Agrícola, 2002. CD-ROM

Valeriano, M.M.; Picini, A.G. Uso de sistemas de informação geográfica para a geração de mapas de médias mensais de temperatura do Estado de São Paulo. Revista Brasileira de Agrometeorologia, Santa Maria, v.8, n.2, p.255-262, 2000.

Ward Jr., J.H. Hierarchical grouping to optimize an objective function. Journal of the American Statistical Association, Alexandria, v.58, n.301, p.236-244, 1963. 